\title{
Cardio-Oncologia no Brasil: Cenário Atual e Perspectivas
}

doi: https://doi.org/10.32635/2176-9745.RBC.2019v65n3.852

\author{
Cardio-Oncology in Brazil: Current Scenario and Perspectives \\ Cardio-Oncología en Brasil: Escenario Actual y Perspectivas
}

Maria Verônica Câmara dos Santos'; Alessandra de Sá Earp Siqueira²; Marcos Jose Pereira Renni³

Com o crescente incentivo às pesquisas e os avanços tecnológicos relacionados à área da oncologia, açóes para diagnósticos cada vez mais precoces e tratamentos mais eficazes vêm proporcionando mais chances de cura e sobrevida a um expressivo número de pacientes com câncer nas diversas faixas etárias. Isso é fruto do advento de novos agentes quimioterápicos, imunoterapia, modalidades de radioterapia mais eficazes e técnicas cirúrgicas mais avançadas. Entretanto, todo esse arsenal terapêutico pode ocasionar sérios efeitos adversos em vários sistemas orgânicos com o potencial de interferir tanto na adequada condução e/ou efetivação do tratamento proposto quanto no prognóstico do paciente após o controle da doença ou cura. As complicaçôes cardiovasculares (cardiotoxicidade), consequentes das diversas alteraçóes do equilíbrio fisiológico do sistema cardiovascular, induzidas pelo tratamento antineoplásico, representam importante parte desse contexto, sendo responsáveis pelas maiores causas de morbimortalidade, após a indesejada evolução do próprio câncer ${ }^{1}$.

O primeiro relato sobre esse assunto ocorreu na década de 1960, mediante observações relacionadas às manifestaçôes clínicas cardiovasculares da daunomicina durante o tratamento de crianças com leucemia. A partir de então, inúmeros estudos clínicos e experimentais, envolvendo todas as faixas etárias, diversos grupos terapêuticos e métodos diagnósticos laboratoriais e de imagem multimodal vêm sendo publicados ${ }^{2}$. A crescente evidência dessas complicaçôes deu margem às organizaçôes de serviços, onde ambas as especialidades começaram a discutir o planejamento terapêutico e suas possíveis consequências no sistema cardiovascular, identificando os pacientes com maior risco ou vulnerabilidade ao seu desenvolvimento - surgiu então o conceito de cardio-oncologia. Nas duas últimas décadas, sociedades nacionais e internacionais foram formadas com reunióes específicas e centros de treinamento criados no sentido de expandir o conhecimento da cardio-oncologia e o aperfeiçoamento de profissionais envolvidos em um espectro multidisciplinar, buscando condiçôes para a continuação do tratamento oncológico com menor risco ou dano cardiovascular ${ }^{2,3}$.

Esses pacientes apresentam oito vezes mais chances de apresentar um desfecho fatal, comparados com a população geral $^{4}$. A insuficiência cardíaca é a complicação mais prevalente, estreitamente relacionada ao uso das antraciclinas, muitas vezes não diagnosticada (subclínica), tornando o prognóstico mais reservado. Outras complicaçóes, também frequentes, são observadas no dia a dia da cardio-oncologia, tais como alteraçóes do endotélio vascular e subsequentes tromboses, hipertensão arterial sistêmica e pulmonar, arritmias, isquemia miocárdica, além de valvulopatias e pericardiopatias ${ }^{5,6}$. O diagnóstico e o manejo dos tumores cardíacos primários e as metástases cardiovasculares provenientes de outros sítios também fazem parte desse grande universo de atuação da cardio-oncologia ${ }^{7}$.

As doenças cardiovasculares e o câncer são as principais causas de morte em países desenvolvidos, com aspectos epidemiológicos e fatores de risco comuns ${ }^{8,9}$. No Brasil, segundo dados do Instituto Nacional de Câncer José Alencar Gomes da Silva (INCA), para cada ano do biênio 2018-2019, a expectativa foi de 600 mil casos novos em adultos e de 12.500 casos novos na população infantojuvenil (0-19 anos). Entre 2009 e 2013, o câncer motivou cerca 12\% dos óbitos na faixa etária infantojuvenil brasileira ${ }^{10}$.

A predisposição genética individual (ainda não rastreada, rotineiramente) e os extremos das idades (jovens e idosos) são também importantes fatores preditivos do desenvolvimento de complicaçôes cardiovasculares. Porém, a maioria desses eventos pode ser precipitada por situaçóes modificáveis e passíveis de atuação com medidas preventivas ou atenuantes,

\footnotetext{
1 Sociedade Brasileira de Oncologia Pediátrica (SOBOPE) - Comitê de Cardio-Oncologia; Sociedade Brasileira de Cardiologia/Departamento de Cardiopatias Congênitas/Cardiologia Pediátrica (DCC/CP-SBC) - Diretoria 2020-21; Sociedade Interamericana de Cardiologia/Grupo Interamericano de Cardio-Oncología Pediátrica (SIAC/GIACOP). Orcid iD: https://orcid.org/0000-0002-4128-6717

${ }^{2}$ Editora-Associada da Revista Brasileira de Cancerologia (RBC) do Instituto Nacional de Câncer José Alencar Gomes da Silva (INCA). Rio de Janeiro (RJ), Brasil. Orcid iD: https://orcid.org/0000-0003-3852-7580

${ }^{3}$ INCA. Rio de Janeiro (RJ), Brasil. Orcid iD: https://orcid.org/0000-0003-3381-7394

Endereço para correspondência: mveronica.santos@uol.com.br
} 
desde que prontamente identificadas. Os fatores de risco mais expressivos são a hipertensão arterial sistêmica, diabetes, tabagismo, sedentarismo, obesidade e consumo excessivo de álcool, todos ambiguamente responsáveis pela maior incidência de doenças cardiovasculares e câncer na população geral, em especial pulmão, mama, próstata e cólon ${ }^{11,12}$.

$\mathrm{O}$ conhecimento e a importância da cardio-oncologia atravessou fronteiras e levantou a necessidade de maior orientação das instituiçóes atuantes no Brasil, quando, em 2011, a Sociedade Brasileira de Cardiologia (SBC) publicou a $1^{a}$ Diretriz Brasileira de Cardio-Oncologia, em adultos e, em seguida, em 2013, a $1^{a}$ Diretriz Brasileira de Cardio-Oncologia Pediátrica, ambas enfatizando a importância dessa área inovadora na prática diária, diante do crescente número de pacientes atendidos em nosso país em todas as faixas etárias ${ }^{13,14}$.

Em novembro de 2016, foi criado o Grupo Brasileiro de Cardio-Oncologia (GBCO), por meio da união de cardiologistas pertencentes à SBC, atuantes na área da cardio-oncologia nacional em várias instituiçôes brasileiras, com o objetivo de compartilhar experiências e auxiliar colegas aspirantes em seus casos clínicos diários pelas redes sociais.

O Brasil segue uma tendência global na implementação da cardio-oncologia como modalidade cardiológica, com crescente adesão por várias instituiçốes acadêmicas e não acadêmicas. $\mathrm{O}$ desenvolvimento de programas de atendimento cardiológico que envolva centros primários de atenção hematológica e oncológica abre perspectivas diretamente ligadas à qualidade da assistência aos pacientes em tratamento de câncer, especialmente diante das possibilidades de complicaçóes cardiovasculares que podem comprometer tanto o andamento do seu protocolo terapêutico como seu prognóstico. A união entre oncologistas e cardiologistas, com o apoio de suas equipes multiprofissionais, favorece o tratamento mais seguro e permite açóes de organização de registros nacionais e programas de atendimento ainda carentes no nosso cenário.

Esta edição da Revista Brasileira de Cancerologia, dedicada à cardio-oncologia, alerta sobre a importância do essencial reconhecimento do crescente número de indivíduos em tratamento ou curados do câncer, levanta a compreensão da necessidade de uma integração multidisciplinar das instituiçôes de tratamento, ensino e pesquisa baseadas na prevenção, monitoramento ativo e intervenção terapêutica em tempo hábil, com o intuito de alcançar o controle desse problema tão sério e permitir uma adequada qualidade de vida aos pacientes ${ }^{15}$.

Boa leitura!

\section{REFERÊNCIAS}

1. Bansal N, Amdani S, Lipshultz ER, et al. Chemotherapy-induced cardiotoxicity in children. Expert Opin Drug Metab Toxicol. 2017;13(8):817-32. doi: https://doi.org/10.1080/17425255.2017.1351547

2. Lenihan DJ, Hartlage G, DeCara J, et al. Cardio-Oncology training: a proposal from the International Cardioncology Society and Canadian Cardiac Oncology Network for a new multidisciplinary specialty. J Card Fail. 2016;22(6):465-71. doi: https://doi.org/10.1016/j.cardfail.2016.03.012

3. Tan C, Tasaka H, Yu KP, et al. Daunomycin, an antitumor antibiotic, in the treatment of neoplastic disease. Clinical evaluation with special reference to childhood leukemia. Cancer. 1967 Mar;20(3):333-53. doi: https://doi. org/10.1002/1097-0142(1967)20:3<333::aid-cncr2820200302>3.0.co;2-k

4. Yeh ETH, Bickford CL. Cardiovascular complications of cancer therapy: incidence, pathogenesis, diagnosis, and management. J Am Coll Cardiol. 2009;53(24):2231-47. doi: https://doi.org/10.1016/j.jacc.2009.02.050

5. Plana JC, Galderisi M, Barac A, et al. Expert consensus for multimodality imaging evaluation of adult patients during and after cancer therapy: a report from the American Society of Echocardiography and the European Association of Cardiovascular Imaging. J Am Soc Echocardiogr. 2014;27(9):911-39. doi: https://doi.org/10.1016/j.echo.2014.07.012

6. Zamorano JL, Lancellotti P, Rodriguez Muñoz D, et al. 2016 ESC position paper on cancer treatments and cardiovascular toxicity developed under the auspices of the ESC Committee for Practice Guidelines: the task force for cancer treatments and cardiovascular toxicity of the European Society of Cardiology (ESC). Eur Heart J. 2016;37(36):2768-2801. doi: https://doi.org/10.1093/eurheartj/ehw211

7. Burke A, Tavora F. The 2015 WHO Classification of tumors of the heart and pericardium. J Thorac Oncol. 2016;11(4):441-52. doi: https://doi.org/10.1016/j.jtho.2015.11.009

8. Siegel R, Naishadham D, Jemal A. Cancer statistics, 2012. CA Cancer J Clin. 2012;62(1):10-29. doi: https://doi. org/10.3322/caac.20138

9. Murphy SL, Xu J, Kochanek KD. Deaths: preliminary data for 2010. Natl Vital Stat Rep. 2012;60(4):1-52.

10. Instituto Nacional de Câncer José Alencar Gomes da Silva. Estimativa 2018: incidência de câncer no Brasil [Internet]. Rio de Janeiro: INCA; 2017. [acesso 2019 set. 22]. Disponível em: http://www1.inca.gov.br/estimativa/2018 
11. Khan N, Afaq F, Mukhtar H. Lifestyle as risk factor for cancer: evidence from human studies. Cancer Lett. 2010;293(2):133-43. doi: https://doi.org/10.1016/j.canlet.2009.12.013

12. Majed B, Moreau T, Senouci K, et al. Is obesity an independent prognosis factor in woman breast cancer?. Breast Cancer Res Treat. 2008;111(2):329-42. doi: https://doi.org/10.1007/s10549-007-9785-3

13. Kalil Filho R, Hajjar LA, Bacal F, et al. I Diretriz Brasileira de Cardio-Oncologia da Sociedade Brasileira de Cardiologia. Arq Bras Cardiol. 2011;96(2 supl 1):1-52.

14. Santos MVC, Paiva MG, Macedo CRDP, et al. I Diretriz Brasileira de Cardio-Oncologia Pediátrica da Sociedade Brasileira de Cardiologia. Arq Bras Cardiol. 2013;100(5 Supl.1):1-68.

15. Scheffer M, coordenador. Demografia Médica no Brasil 2018. São Paulo: FMUSP,CFM, Cremesp; 2018.

Recebido em $28 / 1 / 2020$

Aprovado em $3 / 2 / 2020$ 